Historic, archived document

Do not assume content reflects current scientific knowledge, policies, or practices. 

United States Department of Agriculture di D, Dopurimgut of Agrionltoz Bureau of Biological Survey

Wildlife Research and Management Leaflet BS-29

Washington, D. C.

December 1935

\section{DIRECTIONS FOR PRESERVATION AND CARE OF MATERIAL COLLECTED FOR FOOD HABITS STUDIES}

Prepared in Section of Food Habits, Division of Wildlife Research

\section{Contents}

Page :

Introduction. . . . . . . . . . 1: Preservation of unknown material. . 4 Collection of stomachs. . . . . .2 : Collection of pellets, droppings, Recording data. . . . . . . . .2: and other material. . . . . . 4 Materials needed. . . . . . . . 3 : Shipping . . . . . . . . . 4 Preservatives . . . . . . 3 :

\section{Introduction}

In making studies of the food habits and food preferences of birds, mammals, reptiles, and amphibians, an essential method of approach is through detailed examination of stomach contents. By this means accurate identification of food items is made possible, and likewise some idea of the importance of the various foods can be ascertained through determination of percentages, by bulk, for the items represented. To further these studies there is need for accurate data to accompany stomachs collected and also for practical means of preserving and caring for collections of food material. Many good food habits data have been lost in the past for lack of information on stomach tags, or because of illegibility of the entries. Oversight is sometimes responsible for this but more often it is the result of using unsuitable materials in preparation of stomachs and stomach tags.

Proper methods of handling stomachs and other material collected for food habits studies are given here to help students of the subject and cooperators of the Biological Survey to avoid some of the mistakes that often necessitate the discarding of valuable material. 


\section{Collection of Stomachs}

In removing the stomach (gizzard) of birds, first sever the intestine and if necessary tie with a strong cord. If either gullet or esophagus contains food it should be removed along with the stomach; otherwise it may be cut a short distance from the entrance to the stomach, leaving only a stub for convenience in attaching an identification tag (figs. I and 2). It is important that a crop or gullet containing food be kept with the associated stomach. In the case of reptiles it is well to save the entire digestive tract. This should be folded into more or less symmetrical loops and then enclosed in cheesecloth. It is sometimes impracticable to save the entire stomach contents of deer, elk, moose, and of other large herbivorous mammals. For these it is advisable to open the stomachs and remove about one quart of the contents. If this is taken from the anterior stomach, or rumen, samples should be selected from several points in the mass, or the entire contents thoroughly mixed, before removing the part to be saved. Before doing this, however, the larger and more diagnostic bits of food should be taken out. The sample,along with these larger and more diagnostic fragients can be placed in the cheesecloth bag or wrapping, and then handled in the same manner as a stomach. Some statement as to the actual or approximate quantity of material in such stomachs should be made on the tag or on the schedule.

\section{Recording Data}

The essential data are the name of the animal (scientific name, if known), sex, approximate age (juvenile or adult), - date and hour of day, locality, method of capture (whether shot or trapped, and, if the latter, what kind of bait, if any, was used), and finally the collector's name and number should be carefully recorded for each specimen (fig. 3 ).

Whenever time and opportunity permit, and where special local ecological studies are undertaken, the so-called essential data should be supplemented by detailed notes on all environmental features that are likely to affect food habits. Recording the kinds and abundance of foods available, the nature of the cover, the status of the associated animal species, the weather conditions, and like data will help materially in examining the stomach contents and in interpreting the findings.

A serial, or collector's, number should be assigned to each stomach and the data pertaining to each should be recorded under corresponding series of numbers in a catalogue or on a convenient schedule. If the stomachs are identified only by a serial number on the tag and the data are recorded separately, the numbered tag should also bear the collector's initials (fig. 1). Care should be taken to correlate the information recorded in the schedule (fig. 3) with the number attached to the stomach to which it pertains. In other words, the number on each stomach tag should correspond with a number on the schedule or catalogue. Each collector's numbers should form one continuous series and should not be repeated, regardless of the number of stomachs collected or the periods covered. 
To save time in sorting out miscellaneous lots of stomachs, Biological Survey collectors use letters on stomach tags to designate the various vertebrate groups. The letter "M" for instance prefixes the numbers on all tags attached to mammal stomachs. Similarly, the letter "B" is used to indicate the bird series, and the letter "H" designates amphibian and reptile material. In this system separate series of numbers are used for each group, and the data are recorded in separate catalogues.

Although the recording of essential data on schedules or in catalogues is the accepted procedure, in that data recorded in this manner are more accessible for reierence, the system of noting all data on tags attached directly to stomachs can be employed when necessary (fig. 2). This method is practicable, however, only where small lots of material are concerned.

\section{Materials Needed}

In recording numbers and the collector's initials on stomach tags, a good carbon ink and a good grade of tag should be used. Ordinary inks will fade in preservative solutions. A medium-soft lead pencil is acceptable, but its use should not be considered standard procedure. One surface of the tag may at times become badly soiled, defaced, or bleached, resulting in illegibility of number or initials. As a precaution against loss of data in this manner, the number and initials may be written on one side of the tag in carbon ink and on the other in lead pencil. Labels marked with carbon ink should be permitted to dry thoroughly before being placed in solutions. Tags should be made of a stiff water-resisting paper. Papers having starchy fillers are not suitable, as the surface washes off in water in a very short time. Holes should be punched near one end and threaded with twine suitable for tying tag to stomach.

\section{Preservatives}

The most reliable and economical preservative for stomachs is a formaldehyde solution. Alcohol (70 percent) may be used for the stomachs of small passerine birds, but it is not recommended for the larger species. Medium-sized stomachs can be placed in about a 4 percent formaldehyde solution made by diluting the commercial formaldehyde with 24 parts of water. For the larger stomachs the commercial grade should be diluted with 12 to 15 parts of water, as a stronger preservative is necessary. Large stomachs should be injected with preservative previous to being placed in the formaldehyde solution, or small slits may be made in the stomach wall to insure immediate penetration of the preservative. This may necessitate wrapping the stomach in cheesecloth to prevent leakage of stomach contents.

Stomachs should remain in the preservative for at least 5 days. After that period of fixation they may be shipped safely without spoiling, or they may be left in the preserving solution for indefinite storage.

In removing material from formaldehyde solution it is advisable to use long forceps or rubber gloves to protect the hands, as formaldehyde gas is irritating and tends to dry and shrivel the skin on comparatively short exposure. The preservative is a poison and should be treated as such. 
When the identity of a collected specimen is uncertain, the entire animal, if it happens to be a small species, such as a frog, snake, or mouse, should be preserved. This can be accomplished by injection of a 4 percent formaidehyde solution. For this purpose a glass hypodermic syringe of $5 \mathrm{cc}$ capacity, equipped with needles 1 to 2 inches long is useful. When the preservation of the entire animal is not feasible, the head, feet, and tail may be removed and tied in a piece of cheesecloth along with the stomach before placing in the preservative solution. If the specimen is a bird, the wings should be saved also.

The larger injected specimens should always be immersed in the preservative solution with the injection holes directed upwards to facilitate the rapid escape of any gases that may form before preservation is complete. The collection of these gases within the specimen, in pockets from which they cannot escape, is one of the most frequent causes for the development of decayed spots in improperly preserved material.

\section{Collection of Pellets, Droppings, and Other Material}

Pellets from owls and other birds, mammal droppings, and cheek-pouch material should be collected as individual samples. Examination of individual pellets, for instance, yields more accurate and complete data than bulk or lot analyses. If collected in lots, special attention should be given to packing to insure against disintegration. If this is done the individual pellets can be sorted out at the time of examination. Pellets collected periodically from roosts or nests should always be collected individually and the approximate date of deposition indicated for each. They may be placed in small glassine or paper bags and stored in a dry, insect-proof container. Before being stored, however, all such material should be gassed with carbon disulphide to destroy any of the numerous insect pests that otherwise would soon take possession, and naphthalene flakes should be added to the container. Inspections should be made periodically for signs of insect infestation.

Pellets and droppings should be collected for food habits studies only when the identity of the material is positive. Droppings from known dens or pellets from under known roosts or nests are the most desirable, as certain identification can usually be made in such cases.

\section{Shipping}

When stomachs are to be shipped, or even moved from one locality to another in an automobile, they should be removed from the formaldehyde and permitted to dry until no longer wet on the surface. They may then be wrapped in heayy paper or cloth and placed in a suitable shipping container, such as a tin can or a wooden box lined with a number of layers of newspaper or other absorbent material. Excess space should be filled with crumpled paper or excelsior. The receptacle should then be covered with an outside shipping wrapper, which should be snug and firmly tied. Stomachs or other material sent to the Bureau for food studies should be marked for the attention of the Section of Food Habits, Bureau of Biological Survey, Washington, D. C. 

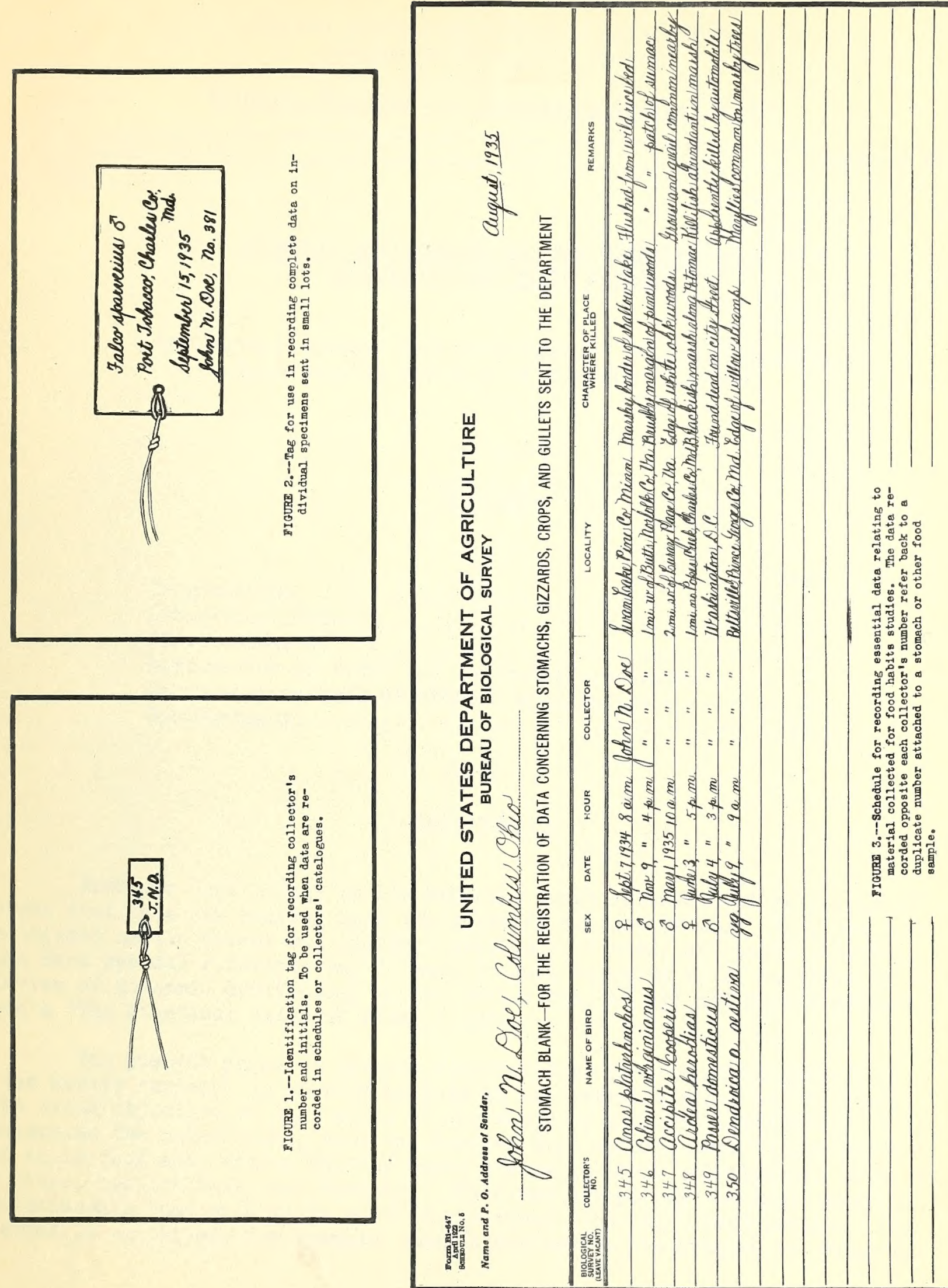


\section{.}

